\title{
Enhancement of Fusarium Head Blight Detection in Single Free-Falling Wheat Kernel Using a Multi- Spectral Inspection System
}

\author{
I-Chang Yang, Ph. D. Candidate
}

Dept. of Bio-Industrial Mechatronics Engineering, National Taiwan University, f90631003@ntu.edu.tw

\section{Stephen R. Delwiche, Agricultural Engineer}

Agricultural Research Service, United States Department of Agriculture, Stephen.Delwiche@ars.usda.gov

\section{Suming Chen, Professor}

Dept. of Bio-Industrial Mechatronics Engineering, National Taiwan University, schen@ntu.edu.tw

\section{Y. Martin Lo, Associate Professor}

Dept. of Nutrition and Food Science, University of Maryland at College Park, ymlo@umd.edu

\author{
Written for presentation at the \\ 2008 ASABE Annual International Meeting \\ Sponsored by ASABE \\ Rhode Island Convention Center \\ Providence, Rhode Island \\ June 29 - July 2, 2008
}

\begin{abstract}
Since the 1800's, Fusarium Head Blight has deleteriously affected the yield and quality of small grain cereal crops such as wheat. This fungal disease is also a health concern due to the frequent production of the secondary metabolite, deoxynivalenol (DON), which is moderately toxic to humans and non-ruminant animals. Our study is a progress report on the effort to develop more efficient methods for separating Fusarium-damaged kernels from sound wheat kernels. Through the development of a high-powered pulsed LED system, we have demonstrated that Fusarium-damaged and sound individual wheat kernels can be correctly categorized at up to 91\% average accuracy. The system is bichromatic in the sense that green and red LEDs are pulsed in sequence, which is similar to our recent work in the past year. In this study, however, the system illumination, signal acquisition, and analysis modules were integrated and improved. Two parameters (slope and $r^{2}$ ) from a regression analysis of the green response onto the red response were used as input parameters in a


linear discriminant analysis (LDA). In agreement with our previous work, slope was the predominant classifier; so much so that it was used exclusively after preliminary analyses. The factors that affect the level of accuracy are the orientation of the optical probe with respect to the LED illumination source that strikes the free-falling kernel, and the color contrast of the two categories, which is observed to vary from sample to sample. Our previous research has shown that commercial highspeed optical sorters are, on average, 50 percent efficient at removing mold-damaged kernels; however, under more carefully controlled, kernel-at-rest, conditions in the laboratory, this efficiency can rise to 95 percent or better. Our present research on free-falling kernels is producing accuracies that are beginning to approach those of the controlled conditions. Knowledge gained from this research will provide design criteria for improvement of high-speed optical sorters for reduction of DON in raw cereal commodities, as well as in finished food products.

Keywords. Fusarium Head Blight, deoxynivalenol, wheat kernel, multispectral inspection

The authors are solely responsible for the content of this technical presentation. The technical presentation does not necessarily reflect the official position of the American Society of Agricultural and Biological Engineers (ASABE), and its printing and distribution does not constitute an endorsement of views which may be expressed. Technical presentations are not subject to the formal peer review process by ASABE editorial committees; therefore, they are not to be presented as refereed publications. Citation of this work should state that it is from an ASABE meeting paper. EXAMPLE: Author's Last Name, Initials. 2008. Title of Presentation. ASABE Paper No. 08----. St. Joseph, Mich.: ASABE. For information about securing permission to reprint or reproduce a technical presentation, please contact ASABE at rutter@asabe.org or 269-429-0300 (2950 Niles Road, St. Joseph, MI 49085-9659 USA). 


\section{Introduction}

Fusarium Head Blight (FHB) has been observed in temperate cereal-producing regions worldwide for more than one hundred years (Stack, 2003), with its prevalence on the rise in recent years. Outbreaks in North America have included those in the early 1980's, the early and mid 1990's, such as in the Midwest in 1996 (Hart, 1998), and lately, the 2003 and 2004 seasons in the soft red winter wheat growing regions of the eastern United States. The mycotoxin, deoxynivalenol (DON), also called vomitoxin, is often produced by this mold, and causes health problems not only to the livestock but also to humans. The regulation of DON in the United States is codified by the Food and Drug Administration's advisory levels (U.S. FDA, 1993), ranging from $1 \mathrm{mg} / \mathrm{kg}$ (human consumption), through $5 \mathrm{mg} / \mathrm{kg}$ (non-ruminant animals), up to 10 $\mathrm{mg} / \mathrm{kg}$ (ruminating beef and chicken) for different product types. Outside the United States, many countries have their similar or more stringent regulations (FAO, 2004), with the European Union setting the human food limit at $0.5 \mathrm{mg} / \mathrm{kg}$ for finished products and $0.75 \mathrm{mg} / \mathrm{kg}$ for flour (Codex, 2003). Aspects of food safety related to DON and tricothecenes in general are reviewed in Dexter and Nowicki (2003). At present, reduction of DON is reliant on methods for removal of kernels afflicted with the fungal disease, and relying upon the positive, albeit imperfect, correlation between the physical damage caused by the disease and the mycotoxin level (Paul et al., 2005).

In recent years, research has been conducted on Fusarium-damaged kernel (FDK) detection based on optical techniques. Two broad categories of optical methods have been explored: digital image analysis and near-infrared spectroscopy. In image studies, the kernel morphology and color characteristics were the primary features used to distinguish damaged from normal kernels (Luo et al., 1999; Ruan et al., 1998). Symptoms of a white or pinkish color and shriveled kernels were caused by the Fusarium damage (Atanasoff, 1920). NIR spectroscopic measurement of DON was initially addressed by Dowell et al. (1999) In NIR spectroscopy studies, Delwiche and colleagues $(2003,2004,2005 a)$ have demonstrated identification of FDK in hard red winter wheat with 95 percent accuracy using two wavelengths, one in the visible region (410-865 $\mathrm{nm}$ ) and the other in the near-IR region (1032-1674 $\mathrm{nm})$. The overall accuracy is nearly as high when two wavelengths are selected from just the visible region.

However, the spectrometer studies were conducted under well-controlled laboratory conditions involving diode array spectrometers and with each kernel at rest. Some recent studies have looked at the potential of high-speed sorting of wheat for intrinsic properties (Paskitan et al., 2003, 2004) or disease (Dowell et al., 2002). An attempt to utilize an equivalent pair of wavelengths in high-speed commercial sorting resulted in an effective reduction of Fusariumdamaged kernel removal efficiency to approximately 50\% of the infected kernels (Delwiche et al. $2005 b)$. As a result of this diminished performance, our recent studies have concentrated on alternative methods for lighting and data collection of free-falling kernels as a possible precursor to an eventual redesign of the optics for high-speed sorting. Based on a design that uses two high-power pulsed LEDs (red and green) as illumination sources, classification accuracy for free-falling Fusarium-damaged kernels has risen to approximately 72 percent, which is better than what was observed for commercial sorting, but shy of the accuracy experienced under controlled laboratory conditions. This has led to redesign considerations for hardware, particularly probe placement, and data analysis.

The objective of the present research is to examine the improvement to Fusarium-damaged kernel recognition under free-falling conditions that arise a) from changes to the orientation of the fiber probe that receives reflected light from the kernel and b) from a modification in the analytical procedure for relating the paired LED responses to one another, which are inherently 
one-half cycle out of phase. Addressing the first change, probe angle is varied from $0^{\circ}$ (the original setting) to $45^{\circ}$; addressing the second, LED pairing during the analysis is changed from a green-after-red (staggered) pairing to an adjusted time equivalent basis through interpolation of the green LED response to the same time interval as the red LED response.

\section{Materials and Methods}

\section{Wheat Kernel Preparation}

Wheat samples originated from a soft red winter wheat breeder's trial set that consisted of Fusarium-inoculated commercial releases or advanced breeders lines, as described in Delwiche (2008). From a pool of 20 retained samples held over from an author's (srd) previous study, 5 samples were selected for the current study. The selection process was based on visual examination of the Fusarium-damaged kernels in comparison to the healthy, normal kernels within the same sample, and making a judgment as to whether the color contrast was high, moderate, or low. From the original pool, two high-contrast, two moderate-contrast, and one low-contrast samples were selected (Fig. 1) for optical tests. For each selection, kernels were drawn at random until equal numbers of 24 normal and 24 Fusarium-damaged kernels were obtained. These 'test' kernels were stored in the wells of immunoassay trays until the time of optical examination. Shown in Figure 1 are representative kernels for each condition (normal, Fusarium-damaged) from each sample, with each kernel displayed in crease down and crease up positions. It is important to realize that a significant challenge for a free-falling optical system is the need to accommodate for the randomness in orientation of the kernel about its long axis, with respect to a viewing probe.

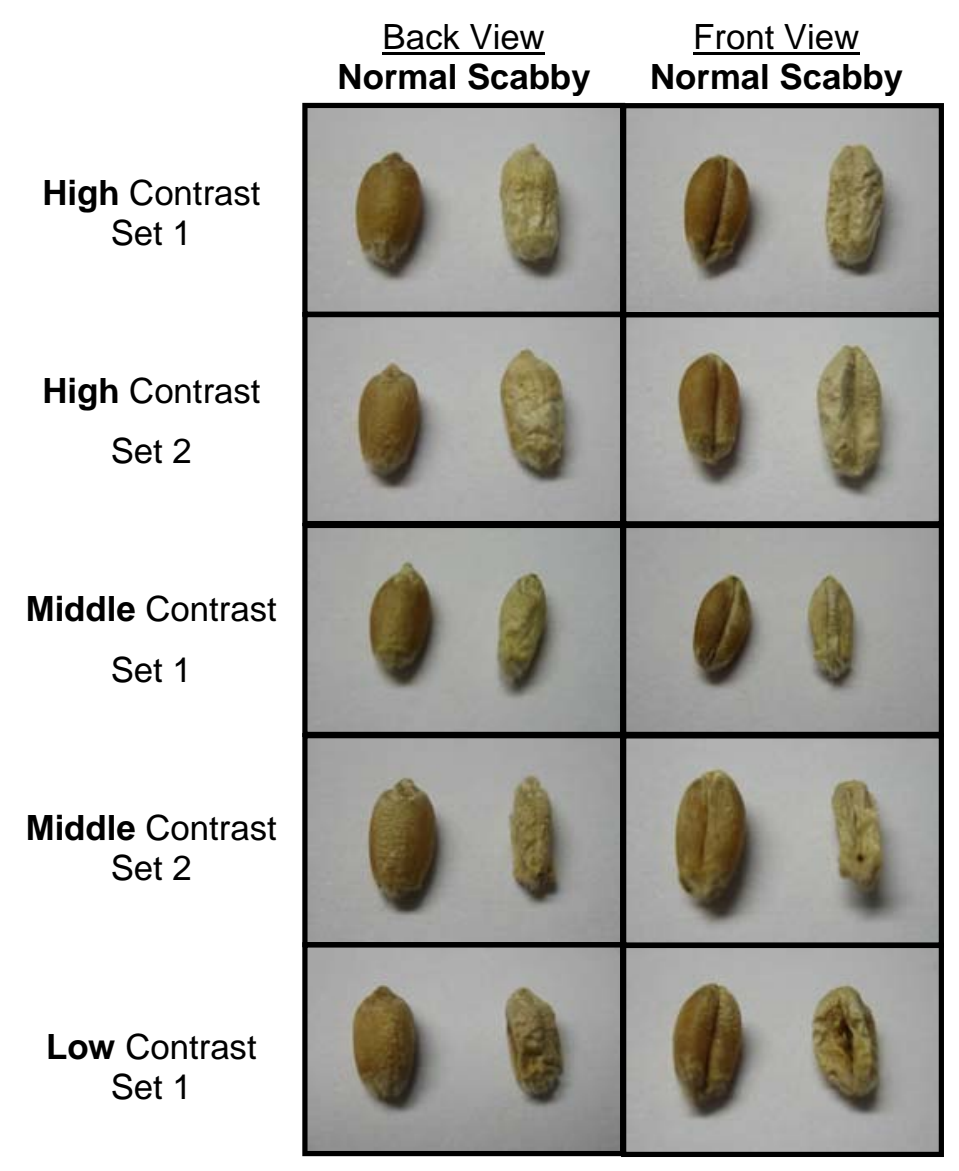


Figure 1 Example of wheat kernel samples for the contrast sets.

\section{Experimental Apparatus and Design}

The sketch of the apparatus for real-time inspection of free-falling wheat kernel inspection system (FFWKIS) is shown in Figure 2. This device consists of a stainless steel tube (914 mm length $\times 6.3 \mathrm{~mm}$ inside diameter (ID)) inclined at a 70 degree angle with respect to the horizontal. The inclination angle of the tube is slightly greater than that of commercial sorters with an open channel, in order to provide a greater gravitation force on the kernel to overcome the friction of the kernel with the tube's interior wall. The lower end of the tube terminates within a plastic slotted open-faced electronics assembly box (76 $\mathrm{mm} \times 67 \mathrm{~mm} \times 35 \mathrm{~mm}$ ) leading to an open-environment inspection region (Fig. 3 left column photos). The box, which is cut away on one end, serves two purposes: as a housing for control electronics and LEDs, and as a guide for receiving the terminal end of the feed tube. Two high-power LEDs [Luxeon I Emitters LXHLPM01 (green) and LXHL-PD01 (red), Phillips Lumileds Lighting Company, San Jose, Calif.] are epoxy-mounted, red-above-green with a center-to-center distance of $16 \mathrm{~mm}$, on a machined Bakelite card that is inserted into one of the box's slots. A small stub of $1 / 2$ inch (OD, outside diameter) stainless steel tubing receives the kernel after it falls past the inspection position. A distance of approximately $30 \mathrm{~mm}$, as measured along a straight line path between the feed and receiver tubes, forms the region for kernel illumination and reflectance capture by an optical fiber bundle.

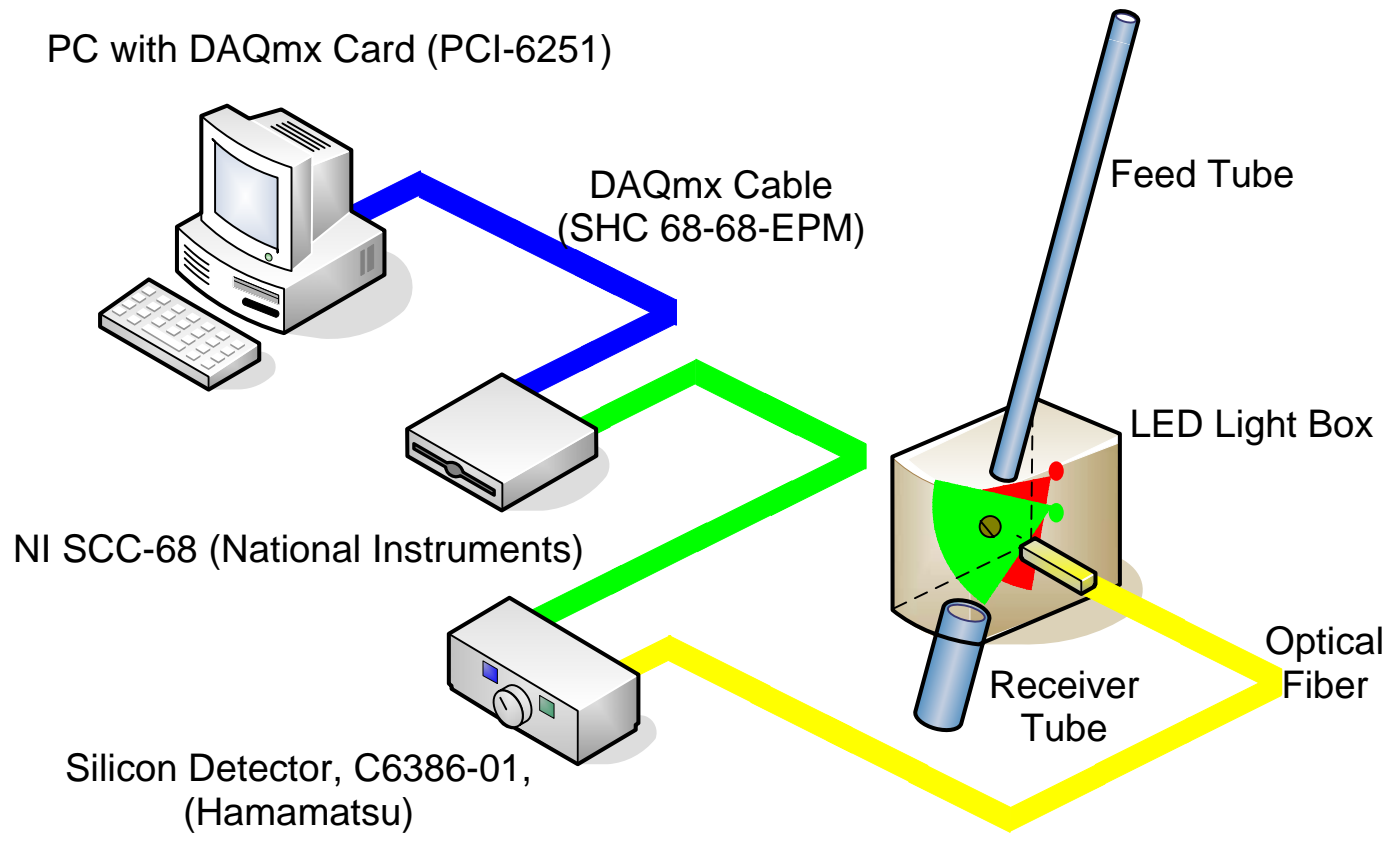

Figure 2 Schematic of the Free-Falling Wheat Kernel Inspection System (FFWKIS) apparatus.

The terminal end of the multi-fiber bundle has an internal diameter of $5 \mathrm{~mm}$ and is oriented in one of four angular positions $\left(0^{\circ}, 15^{\circ}, 30^{\circ}\right.$, and $\left.45^{\circ}\right)$ with respect to the mounting surface of the two illumination LEDs. The fixture holding the end of the bundle can be adjusted for different 
angles. The opposite end of the multi-fiber bundle is connected by SMA connector to a silicon detector/amplifier assembly (Hamamatsu, Model C6386-01). The analog signal from this amplifier is digitized and stored by the inspection system with the use of a data grabber (NIDAQmx PCl-6251, National Instruments), connector (NI SCC-68, National Instruments), and cable (SHC 68-68-EPM) (Fig.2).

The inspection system originally employed LED illumination of the kernel perpendicular to the angle of reflectance measurement by the optical fiber bundle (i.e., the $0^{\circ}$ position). For the purpose of enhancing the accuracy of the system, kernel inspection was conducted using the three additional angles of the optical fiber bundle (Figure 3 ), beyond the original $0^{\circ}$ position. From the top view in Figure 4, it can be seen that changing the angle of the optical fiber can theoretically provide stronger surface reflectance signal intensity.

The side view in Figure 5 shows that the direction of the free-falling kernel primarily along the common centerlines of the feed and receiving tubes, such that the light from the red and green LEDs overlapped mostly on one side of the kernel.
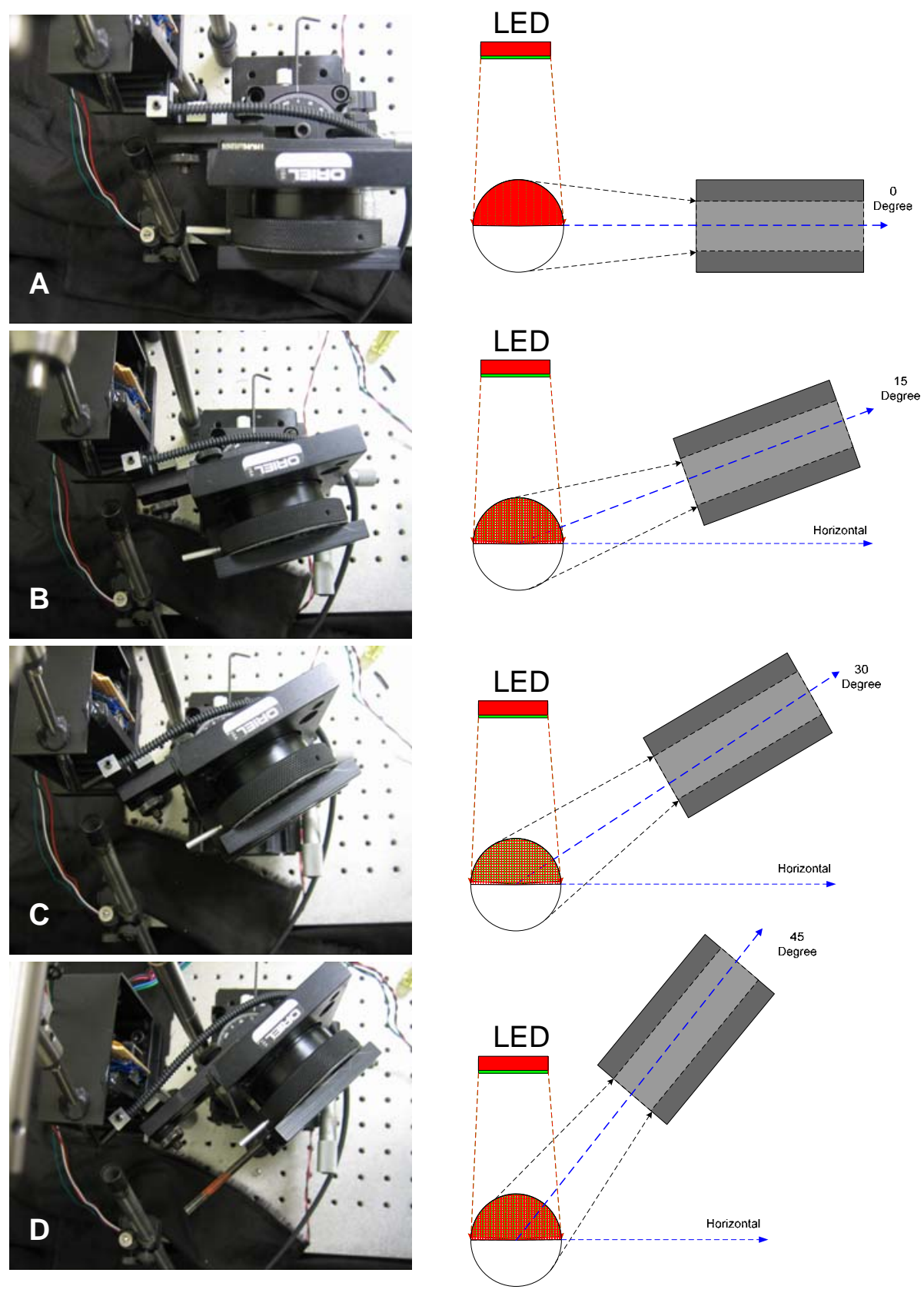
Figure 3 Top view of wheat kernel illumination and spectral measurement in the FFWKIS.

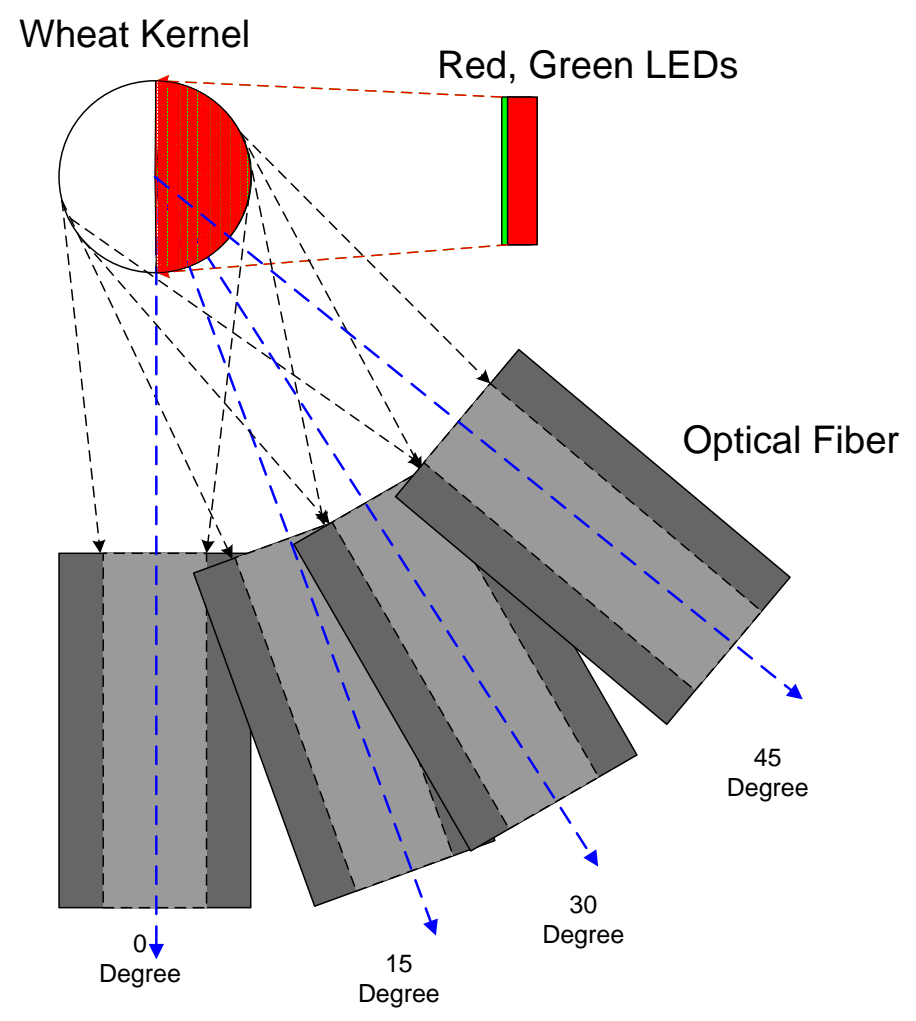

Figure 4 Comparison of equidistant spectral measurements acquired at the four angles.

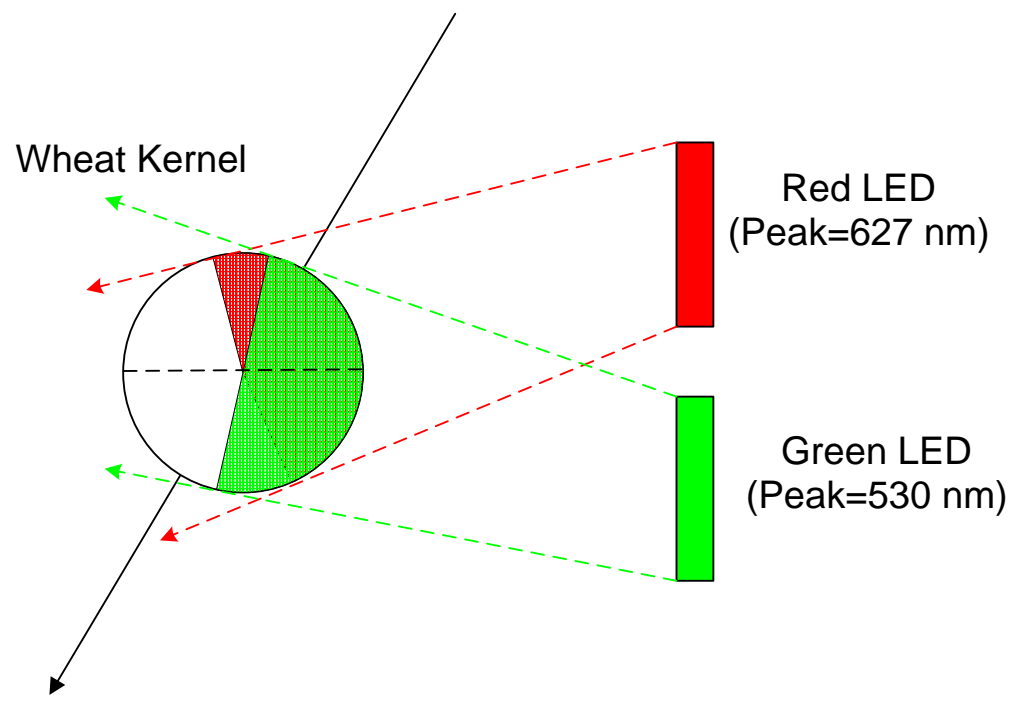

Falling Direction

Figure 5 Side view of the wheat kernel illumination in the FFWKIS. 


\section{Operation}

A 25-ms data collection period with $2 \mathrm{KHz}$ LED alternating frequency is used for the inspection of each kernel. Each of two high power LEDs is turned on for one half cycle, with the red and green LEDs alternating in sequence. The illumination time for an LED, which is also its rest time in the next half cycle, is $0.25 \mathrm{~ms}$. In comparison with the previous studies, the $8 \mathrm{KHz}$ sampling frequency decreased the scans per half cycle to 2 (Fig.6), from the 10 scans per half cycle used in the previous studies. A free-falling wheat kernel inspection system software program (Fig.7) was written using LabVIEW 8.2 (National Instruments, Austin, TX). This single program is used to control the pulsing of the LEDs, as well as to capture the detector signal in synchronization with the LEDs. Signal acquisition is triggered by a $20 \mathrm{mV}$ window trigger with 40 pre-trigger data points. An algorithm was developed to determine the full width at half maximum (FWHM) of the time domain response of reflected light from each of the two LEDs, as the kernel traverses the path of the probe's field of view. The purpose of this algorithm is to establish the baseline signal of the response detector. With baselines established, the green LED's time domain response is approximated by a cubic spline interpolation function, whereupon a pair of reading values is estimated for the green LED at the same time instances as the two red LED response in the preceding half-cycle. This procedure is a departure from our previous study (Delwiche, 2008), in which the green half cycle was directly paired with the preceding red half cycle, without interpolation to a common time instant.

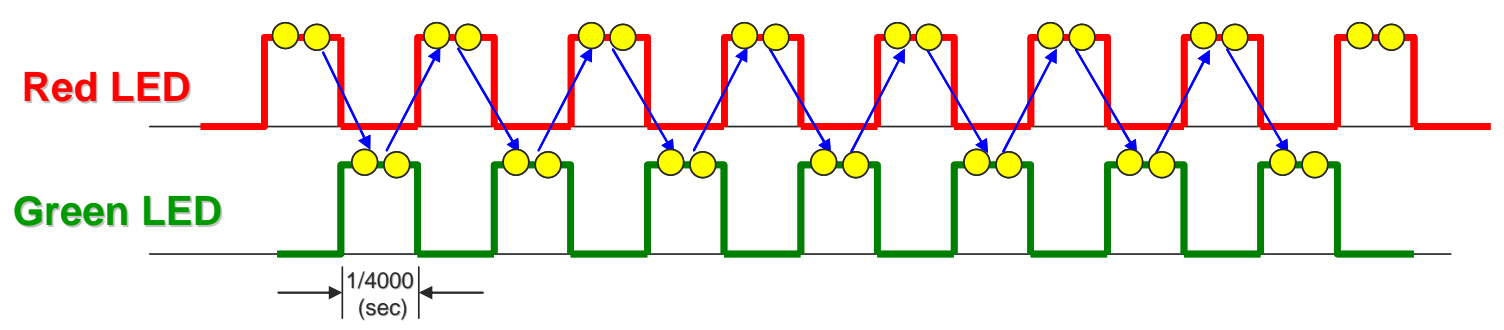

Figure 6 The half cycles of the green and red LEDs are presented. The yellow dots represent the sampling performed by the data acquisition card.

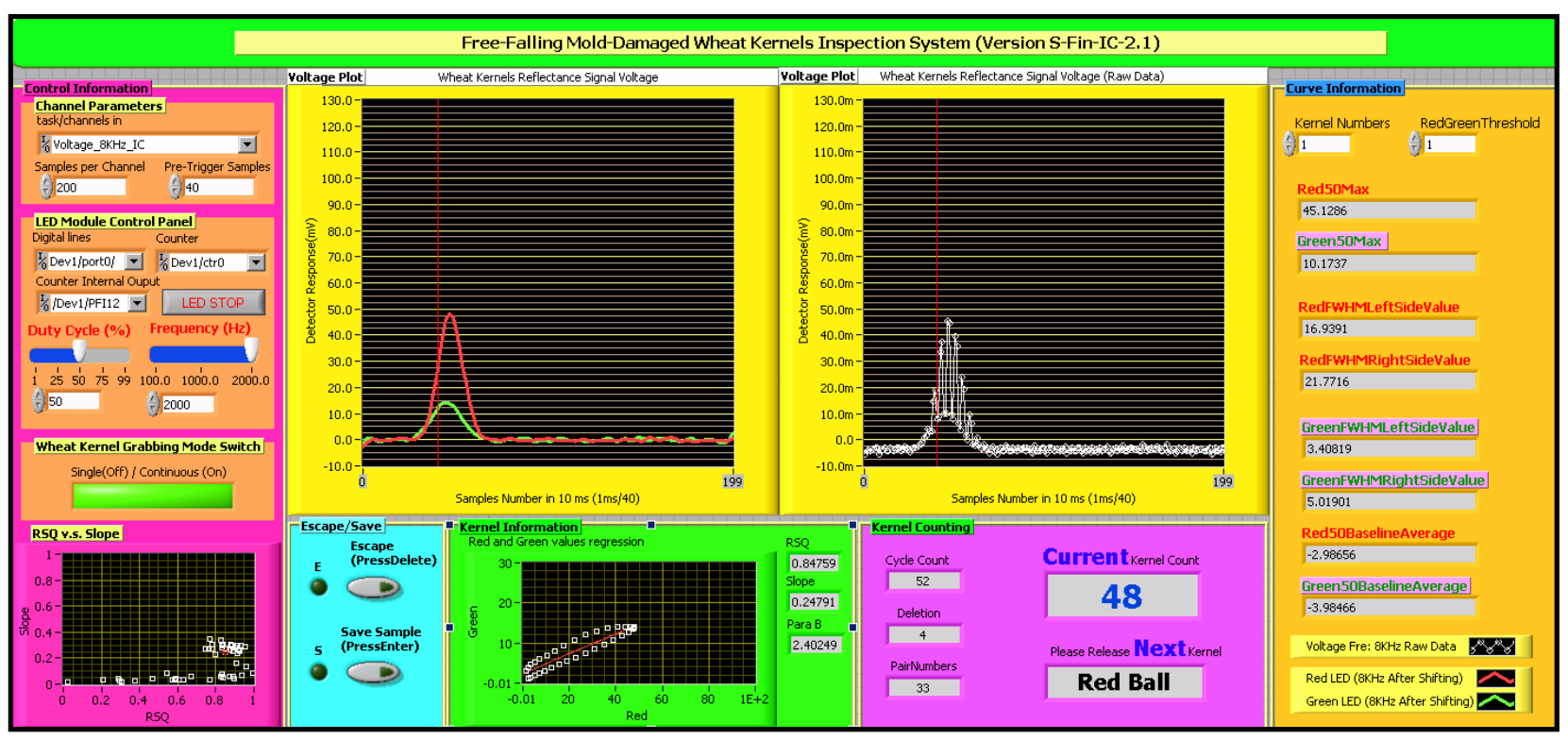


Figure 7 The software interface of the Free-Falling Wheat Kernel Inspection System.

\section{Data Analysis}

The speed of the falling kernel was greatly influenced by the interaction of the kernel with the tube wall, such that some kernel traces demonstrated that the kernel was in the probe's field of view for a small portion of the 25-ms data collection window, while other kernels used nearly the entire window. The collection of off-baseline $(>1.5 \mathrm{mv})$ response pairs of red vs. green reflectance signals for each kernel was the basis for development of classifying the kernel into the normal or scab-damaged category. From this set of paired responses for each kernel, the green light responses were linearly regressed onto the red light responses. The data reduction procedure was performed within LabVIEW immediately after data collection. The results of the regression, particularly the slope and coefficient of determination as well as the paired response readings, were stored to an external file for cluster analysis at a later time.

Only non-baseline values for red- and interpolated green-LED responses were used in regression analysis, according to the following procedure. The peak distance (peak - baseline) was separately determined for the left and right sides of the waveform. Left and right vertical distances, rather than just one distance calculation, were determined for the purpose of accounting for the occasional incidence of a slanted baseline. For each vertical distance, a horizontal distance (i.e., along the time axis) was determined that corresponded to the halfmaximum point $\left(H W H M_{L}\right.$ and $\left.H_{W H M}\right)$. Based on trial and error, a horizontal cutoff distance of $2.2 \times \mathrm{HWHM}$ was established for left and right sides. Readings lying outside of the left and right cutoffs were excluded from regression analysis, as were points lying within the central acceptance region but less than $1.5 \mathrm{mV}$ in magnitude. This procedure was implemented to enhance the reliability of the regression analysis of the waveform readings.

From our earlier study (Delwiche, 2008), two results from the regression analysis - the slope of the line of best fit and the coefficient of determination $\left(r^{2}\right)$ - were examined as input parameters in a linear discriminant analysis (LDA) model (Fig.8) for correlating the relationship between the two visible wavelength (red and green) reflectances to the color variation between the normal and Fusarium-damaged kernel. The coefficient of determination, in contrast, was postulated to be sensitive to surface textural differences between kernel classes. Given these hypotheses, one would expect that the coefficient of determination would have a greater role in classification of normal vs. Fusarium-damaged wheat, compared to red vs. white wheat. Classification accuracy was reported as the percentages of kernels in each category correctly classified, based on a leave-one-out cross validation. Data reduction and classification analyses were performed in the SAS (Version 9.1) environment, using the procedure, 'Discrim', for the LDA (SAS, 2004). Based on the results of the earlier study, it was found that slope was the predominant classifier, with the coefficient of determination adding only a marginal improvement. Therefore, in the present study, only slope was used in the cluster analysis.

Two clustering procedures were used. The first procedure involved the systematic trial of a value for a slope threshold, choosing a value between 0 and 1 in increments of 0.001 , then determining the number of normal and Fusarium-damaged kernels lying on opposite sides of the threshold. From this stepwise search, the optimal threshold was determined for each sample of 24 normal and 24 Fusarium-damaged kernels. The second procedure was similar to our earlier study involving linear discriminant analysis, this time with resubstitution only (i.e., no cross-validation because of similarity of findings - see Results section). The slope threshold procedure serves as an upper bound to the accuracies that can be obtained from the LDA procedure. Programming of the first procedure was performed in MATLAB 6.5 (The Mathworks, 
Inc., Natick, MA); the second procedure was performed in SAS. The results from the regression analysis were also analyzed using MATLAB 6.5 to calculate the optimal threshold of the slope without the coefficient of determination $\left(r^{2}\right)$. Analyses of variance were performed on both procedures, in which the total percent correct was tested with probe angle as the main factor and contrast group as a blocking factor. Comparisons of means were performed using Duncan's multiple range test.

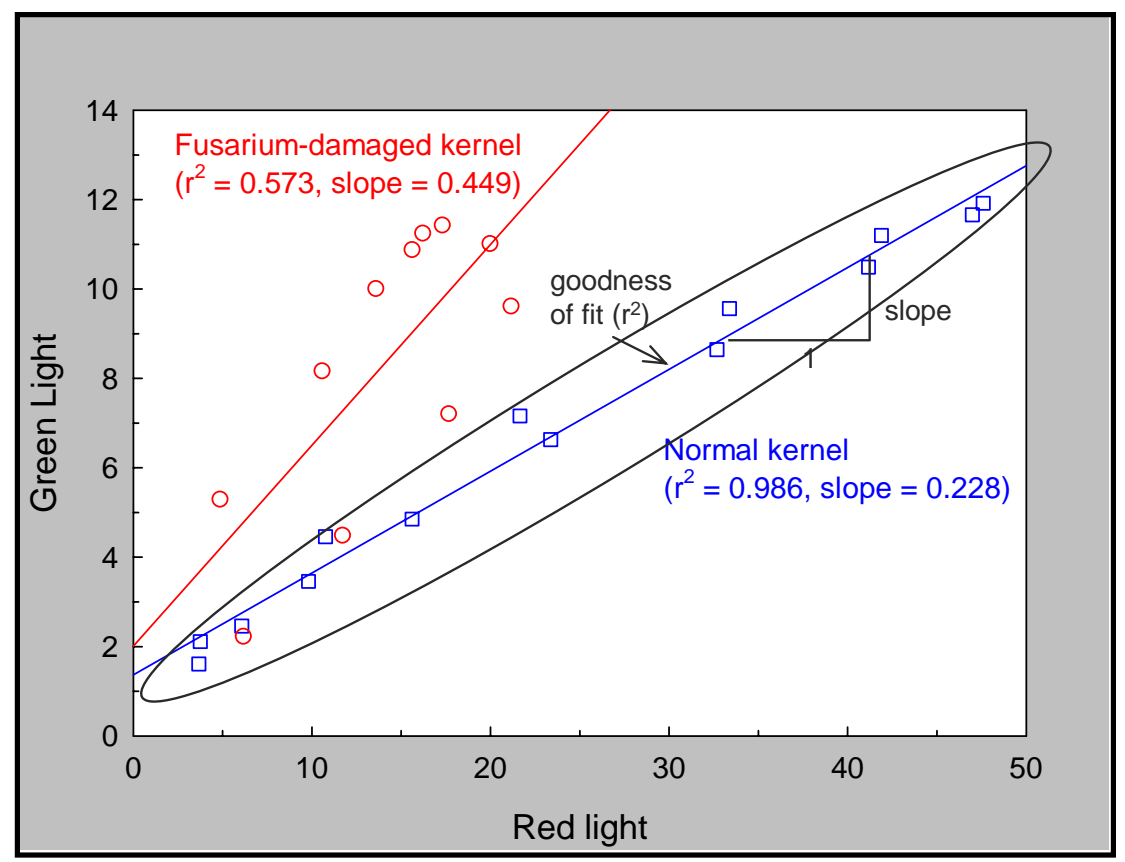

Figure 8 The concept of linear discriminant analysis for separating normal and Fusariumdamaged kernels.

\section{Results and Discussion}

\section{Signal Acquisition}

Figure 9 shows an example of all the kernel responses for one of the trays in the high-contrast set, inspected at the $45^{\circ}$ measurement angle, and subsequently displayed in the MATLAB 6.5 environment. The odd-numbered samples are the normal kernels, and the even-numbered samples are the Fusarium-damaged kernels. Generally, the traces of the normal kernels tend to demonstrate a narrower and higher Gaussian-shaped time response compared to the Fusarium-damaged kernels, which produced the lower and wider responses. The narrower waveform is indicative of a higher speed during freefall caused by the greater mass of normal kernels which results in a smaller influence of air resistance. Because the kernel is inspected in free-fall, having first tumbled through the feed tube, it is not possible to precisely align the kernel about its long axis. Therefore, it is advantageous for robust classification algorithms to be uninfluenced by the rotational position of the kernel. 


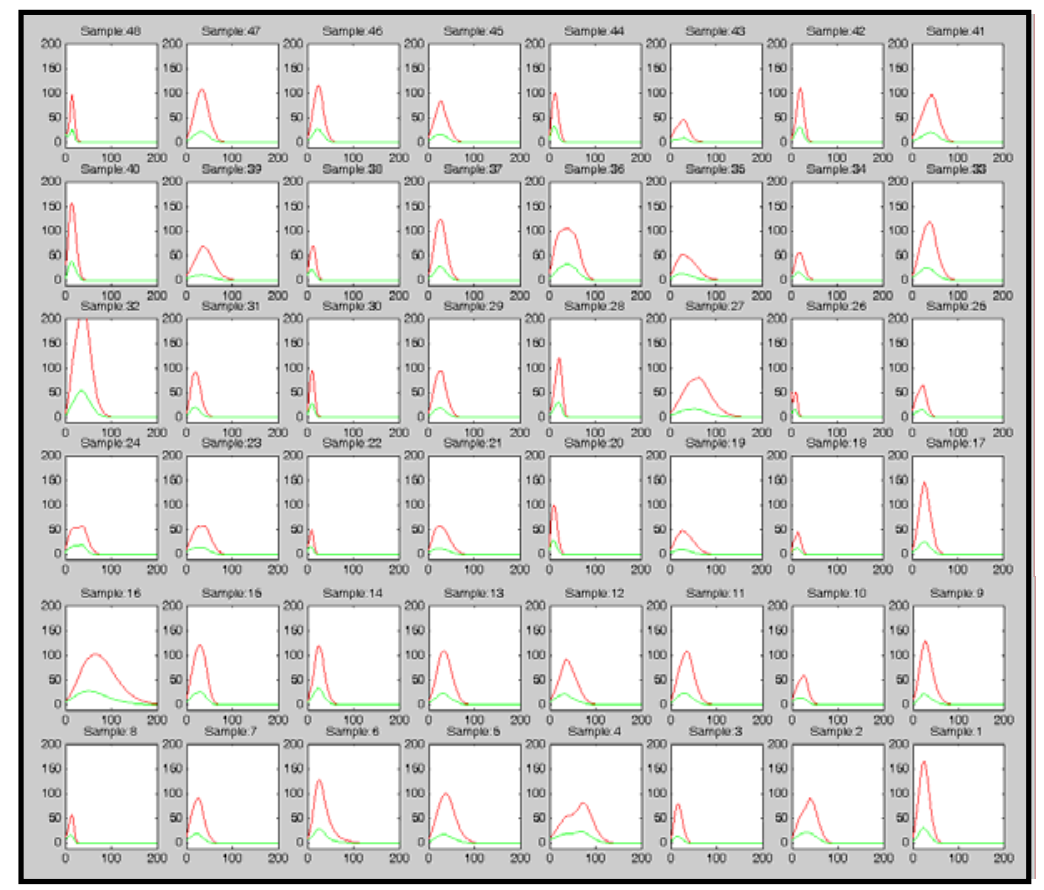

Figure 9 The reflectance response curves of 48 kernels in one tray. The curves of the kernels in high contrast set 1 , inspected with a 45-degree optic fiber angle, are shown as an example.

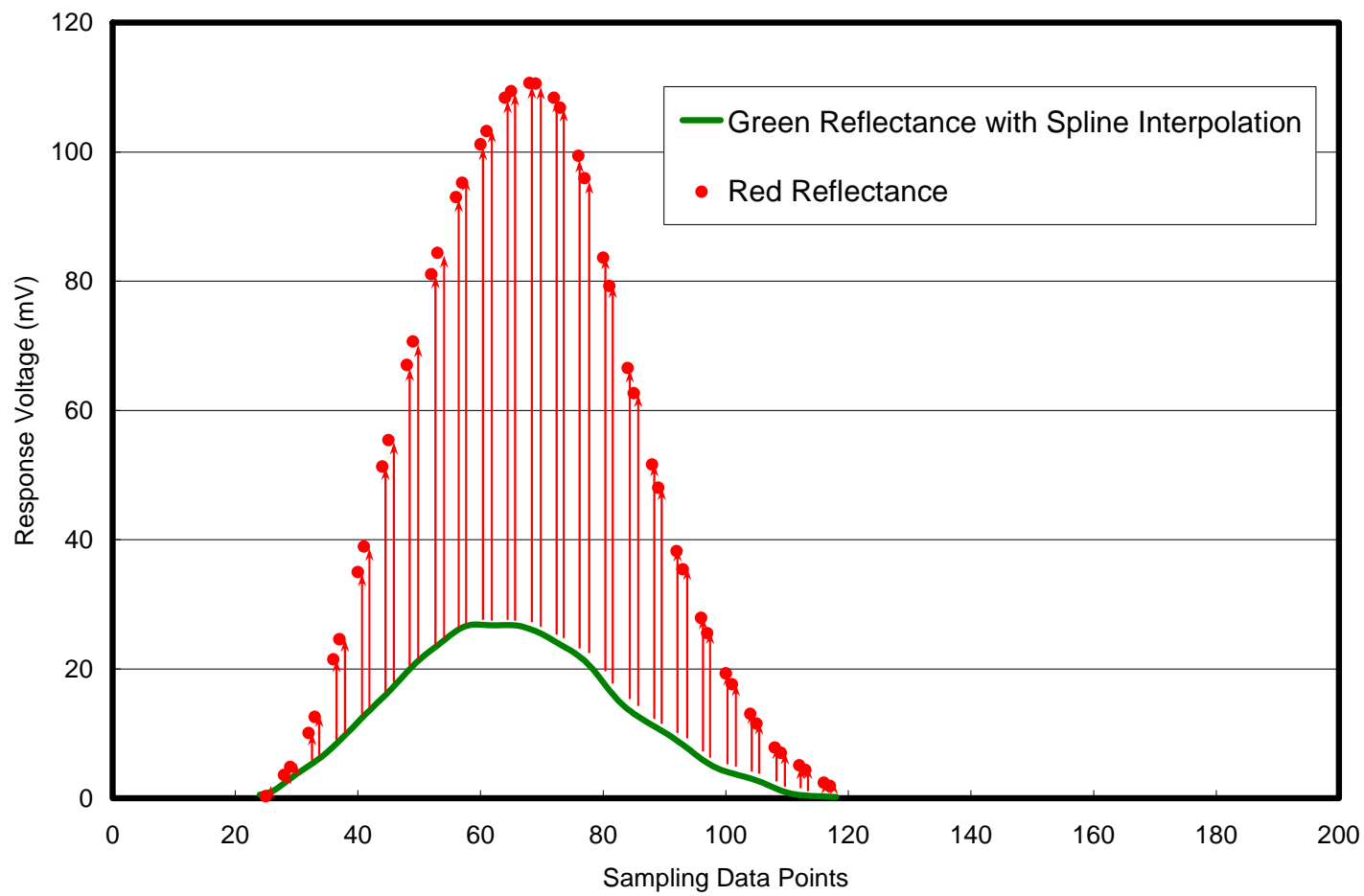

Figure 10 Close-up plot of the red reflectance measurements and the spline interpolation of the green reflectance measurements for kernel number 33 in high contrast set 1. 
The reflectance curves of a typical kernel after green response interpolation is shown in Figure 10. Recall that there were two readings for each half cycle. Prior to the interpolation, the fullwidth at half maximum (FWHM) definition was applied to both LED responses, for the purpose of establishing a baseline. Having defined the FWHM of each LED's waveform, readings at more than 2.2 times the half width from the center line (typically $<1.5 \mathrm{mV}$ ), as well as central region readings $<1.5 \mathrm{mV}$, were discarded from the regression analysis. The algorithm of FWHM was developed to avoid the effect of a sloping baseline.

\section{Angle and Analysis Methods}

The schematic of Figure 4 shows the areas of the kernel covered by the four different probe angles. The intensity of the reflected energy from the kernel was greatly affected by the probe angle. Whereas the peak response of the red LED was typically 50 to $100 \mathrm{mV}$ when the probe angle was $0^{\circ}$, this value rose to approximately $250 \mathrm{mV}$ at the $45^{\circ}$ angle. The green LED response, always smaller than the red LED response, also increased as the angle moved from $0^{\circ}$ to $45^{\circ}$.

According to the aerodynamic property of the free-falling kernel and the random interaction of the kernel and feed tube wall, the kernel can easily be affected by the frictional forces within the tube. The kernel falls in varying direction, albeit confined within the narrow tube, such that its exit speed and rotational position are also variable. Therefore, the $r^{2}$ value is variable in both Fusarium-damaged and normal situations. Preliminary LDA trials (not shown) that utilized both slope and $r^{2}$ as classification parameters failed to demonstrate the added benefit of the latter parameter. Thus, we surmise that the effect of the randomness of kernel orientation on reflected energy was sufficiently large to overshadow any effect that might have been attributed to surface texture differences between normal and Fusarium-damaged kernels. For this reason, only the slope was used in the cluster analysis.

Using the slope parameter in LDA, the classification results, as defined by resubstitution of each sample's reading into the LDA model, are shown in Table 1. The best total result was 91.67\%, for the $2 \mathrm{H}$ set under the $45^{\circ}$ condition, with 21 of 24 and 23 of 24 Fusarium-damaged and normal kernels correctly classified, respectively. The corresponding cross-validation (based on a one-kernel-out per rotation cycle design) accuracies for this set were the same as the resubstitution accuracies, which was also true for 17 of the 19 other set $\times$ angle conditions. Because of the similarity in resubstitution and cross-validation accuracies, the latter values are hereafter not reported. Using another set $\left(1 \mathrm{H} \times 45^{\circ}\right)$ as an example, a plot of slope vs. $r^{2}$ for the 24 Fusarium-damaged and 24 normal kernels of this sample is shown in Figure 11, in which 24 and 20 correct classifications were respectively realized, yielding a total accuracy of $91.67 \%$. The corresponding total accuracy for the LDA was $87.50 \%$. The results of the optimal slope threshold calculation for all sets are shown in Table 2, which is arranged in the same manner as the LDA results of Table 1.

Both Table 1 (LDA results) and Table 2 (thresholding) show the effect of probe angle on the classification accuracy. For either procedure, the $45^{\circ}$ probe angle was significantly different $(P$ $=0.05$ ) than the $0^{\circ}$ angle, with $15^{\circ}$ and $30^{\circ}$ angle accuracies lying between the two extremes. This finding is indicative of the benefit of positioning the probe at an oblique angle with respect to the illumination path, as opposed to the $0^{\circ}$ angle used in our previous free-falling studies. 
Table 1 Results of Linear Discriminant Analysis (LDA) for all measurement angles and contrast sets. Nonsignificant differences among the average values for the total percent correct at the four angles are indicated by common letter $(P=0.05)$ in the rightmost column.

\begin{tabular}{|c|c|c|c|c|c|c|c|}
\hline \multirow[t]{2}{*}{ Angle } & \multirow{2}{*}{$\begin{array}{l}\text { Contrast } \\
\text { Level }\end{array}$} & \multicolumn{2}{|c|}{$\begin{array}{l}\text { Correctly Identified } \\
\text { Scab }\end{array}$} & \multicolumn{2}{|c|}{$\begin{array}{c}\text { Correctly Identified } \\
\text { Normal }\end{array}$} & \multicolumn{2}{|c|}{ Total Correct } \\
\hline & & Out of 24 & (\%) & Out of 24 & (\%) & Out of 48 & $(\%)$ \\
\hline \multirow{5}{*}{0} & $1 \mathrm{H}$ & 19 & 79.17 & 18 & 75.00 & 37 & 77.08 \\
\hline & $1 \mathrm{~L}$ & 16 & 66.67 & 16 & 66.67 & 32 & 66.67 \\
\hline & $1 \mathrm{M}$ & 15 & 62.50 & 16 & 66.67 & 31 & 64.58 \\
\hline & $2 \mathrm{H}$ & 17 & 70.83 & 19 & 79.17 & 36 & 75.00 \\
\hline & $2 \mathrm{M}$ & 18 & 75.00 & 19 & 79.17 & 37 & 77.08 \\
\hline \multirow{5}{*}{15} & $1 \mathrm{H}$ & 20 & 83.33 & 20 & 83.33 & 40 & 83.33 \\
\hline & $1 \mathrm{~L}$ & 15 & 62.50 & 18 & 75.00 & 33 & 68.75 \\
\hline & $1 \mathrm{M}$ & 19 & 79.17 & 24 & 100.00 & 43 & 89.58 \\
\hline & $2 \mathrm{H}$ & 18 & 75.00 & 20 & 83.33 & 38 & 79.17 \\
\hline & $2 \mathrm{M}$ & 18 & 75.00 & 21 & 87.50 & 39 & 81.25 \\
\hline \multirow{5}{*}{30} & $1 \mathrm{H}$ & 20 & 83.33 & 21 & 87.50 & 41 & 85.42 \\
\hline & $1 \mathrm{~L}$ & 17 & 70.83 & 20 & 83.33 & 37 & 77.08 \\
\hline & $1 \mathrm{M}$ & 16 & 66.67 & 18 & 75.00 & 34 & 70.83 \\
\hline & $2 \mathrm{H}$ & 19 & 79.17 & 22 & 91.67 & 41 & 85.42 \\
\hline & $2 \mathrm{M}$ & 17 & 70.83 & 17 & 70.83 & 34 & 70.83 \\
\hline \multirow{5}{*}{45} & $1 \mathrm{H}$ & 20 & 83.33 & 22 & 91.67 & 42 & 87.50 \\
\hline & $1 \mathrm{~L}$ & 18 & 75.00 & 22 & 91.67 & 40 & 83.33 \\
\hline & $1 \mathrm{M}$ & 19 & 79.17 & 19 & 79.17 & 38 & 79.17 \\
\hline & $2 \mathrm{H}$ & 21 & 87.50 & 23 & 95.83 & 44 & 91.67 \\
\hline & $2 \mathrm{M}$ & 21 & 87.50 & 20 & 83.83 & 41 & 85.42 \\
\hline
\end{tabular}

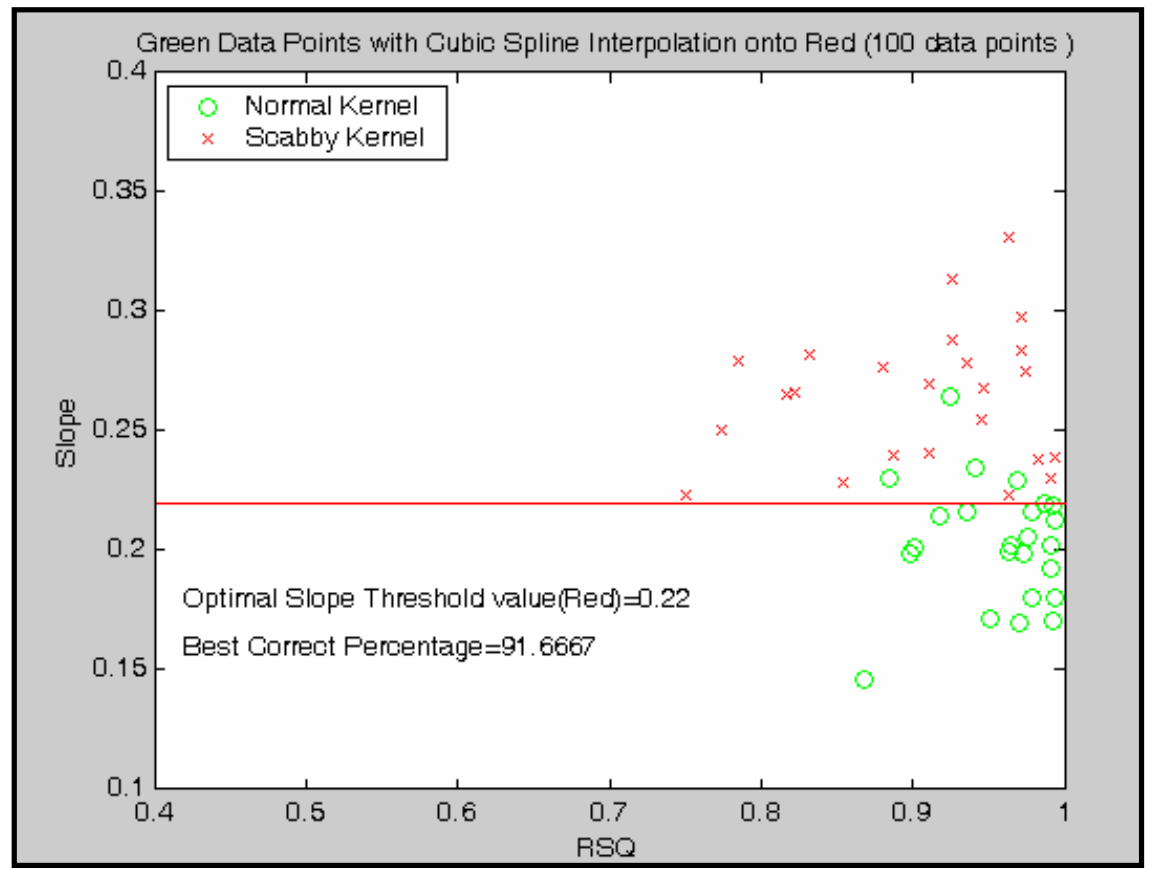


Figure 11 The optimal threshold for separating the normal and Fusarium-damaged kernels for one tray (high contrast set 1 ) at a $45^{\circ}$ fiber probe angle.

Table 2 Results of the optimal threshold method for all measurement angles and contrast sets. Nonsignificant differences among the average values for the total percent correct at the four angles are indicated by common letter $(P=0.05)$ in the rightmost column.

\begin{tabular}{|c|c|c|c|c|c|c|c|c|}
\hline \multirow[t]{2}{*}{ Angle } & \multirow[t]{2}{*}{ Contrast Level } & \multirow{2}{*}{$\begin{array}{l}\text { Slope } \\
\text { Threshold }\end{array}$} & \multicolumn{2}{|c|}{$\begin{array}{l}\text { Correctly Identified } \\
\text { Scab }\end{array}$} & \multicolumn{2}{|c|}{$\begin{array}{l}\text { Correctly Identified } \\
\text { Normal }\end{array}$} & \multicolumn{2}{|c|}{ Total Correct } \\
\hline & & & Out of 24 & (\%) & Out of 24 & (\%) & Out of 48 & (\%) \\
\hline \multirow{5}{*}{0} & $1 \mathrm{H}$ & 0.249 & 20 & 83.33 & 18 & 75.00 & 38 & 79.17 \\
\hline & $1 \mathrm{~L}$ & 0.305 & 13 & 54.17 & 21 & 87.50 & 34 & 70.83 \\
\hline & $1 \mathrm{M}$ & 0.262 & 16 & 66.67 & 15 & 62.50 & 31 & 64.58 \\
\hline & $2 \mathrm{H}$ & 0.269 & 17 & 70.83 & 20 & 83.33 & 37 & 77.08 \\
\hline & $2 \mathrm{M}$ & 0.269 & 18 & 75.00 & 19 & 79.17 & 37 & 77.08 \\
\hline \multirow{5}{*}{15} & $1 \mathrm{H}$ & 0.271 & 21 & 87.50 & 20 & 83.33 & 41 & 85.42 \\
\hline & $1 \mathrm{~L}$ & 0.303 & 14 & 58.33 & 21 & 87.50 & 35 & 72.92 \\
\hline & $1 \mathrm{M}$ & 0.267 & 19 & 79.17 & 24 & 100.00 & 43 & 89.58 \\
\hline & $2 \mathrm{H}$ & 0.264 & 21 & 87.50 & 20 & 83.33 & 41 & 85.42 \\
\hline & $2 \mathrm{M}$ & 0.257 & 23 & 95.83 & 19 & 79.17 & 42 & 87.50 \\
\hline \multirow{5}{*}{30} & $1 \mathrm{H}$ & 0.250 & 20 & 83.33 & 21 & 87.50 & 41 & 85.42 \\
\hline & $1 \mathrm{~L}$ & 0.233 & 22 & 91.67 & 18 & 75.00 & 40 & 83.33 \\
\hline & $1 \mathrm{M}$ & 0.250 & 17 & 70.83 & 18 & 75.00 & 35 & 72.92 \\
\hline & $2 \mathrm{H}$ & 0.246 & 19 & 79.17 & 23 & 95.83 & 42 & 87.50 \\
\hline & $2 \mathrm{M}$ & 0.263 & 17 & 70.83 & 18 & 75.00 & 35 & 72.92 \\
\hline \multirow{5}{*}{45} & $1 \mathrm{H}$ & 0.220 & 24 & 100.00 & 20 & 83.33 & 44 & 91.67 \\
\hline & $1 \mathrm{~L}$ & 0.240 & 20 & 83.33 & 22 & 91.67 & 42 & 87.50 \\
\hline & $1 \mathrm{M}$ & 0.242 & 21 & 87.50 & 18 & 75.00 & 39 & 81.25 \\
\hline & $2 \mathrm{H}$ & 0.223 & 23 & 95.83 & 22 & 91.67 & 45 & 93.75 \\
\hline & $2 \mathrm{M}$ & 0.246 & 20 & 83.33 & 22 & 91.67 & 42 & 87.50 \\
\hline
\end{tabular}

\section{Contrast of Kernels}

The contrast levels of the different kernel sets were selected by visual observation, with the visible subjective criteria and without specialized scientific experience. Basically, the determination of the contrast sets depended on the contrast of kernel color as recognizable by the human eye. The middle contrast sets had fewer highly noticeable damaged kernels and the lower contrast set had only subtle visual differences between normal and Fusarium-damaged kernels. There were no apparent differences in overall kernel size (i.e., length and width) among the five samples. When averaged across angular position, the two high contrast sets produced higher accuracies than the other sets. The accuracies of both high contrast sets were able to reach over $90 \%$ in different analysis methods. For the effect of angle and the contrast, the results of 15 and 30 degree are variable with middle contrast sets (Fig.12). 


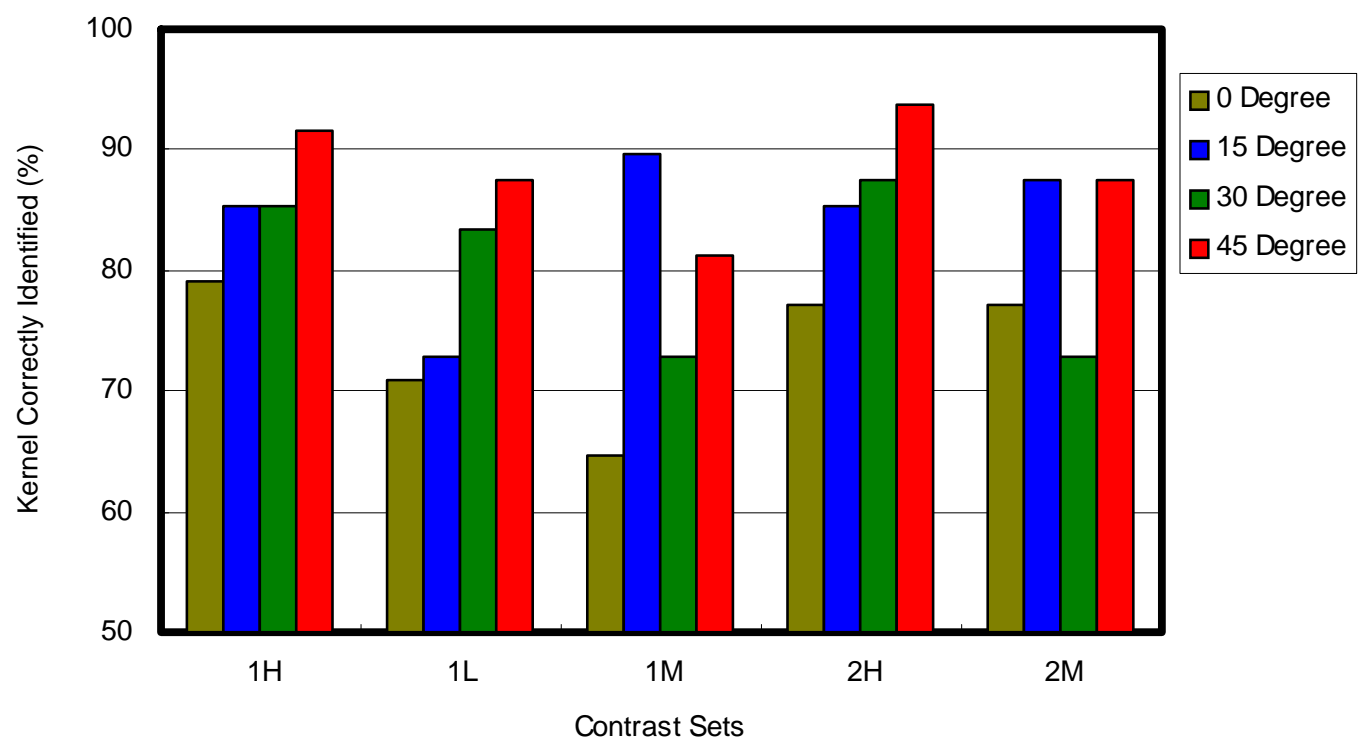

Figure 12 Kernels correctly classified using the optimal threshold determined for each angle and contrast sets.

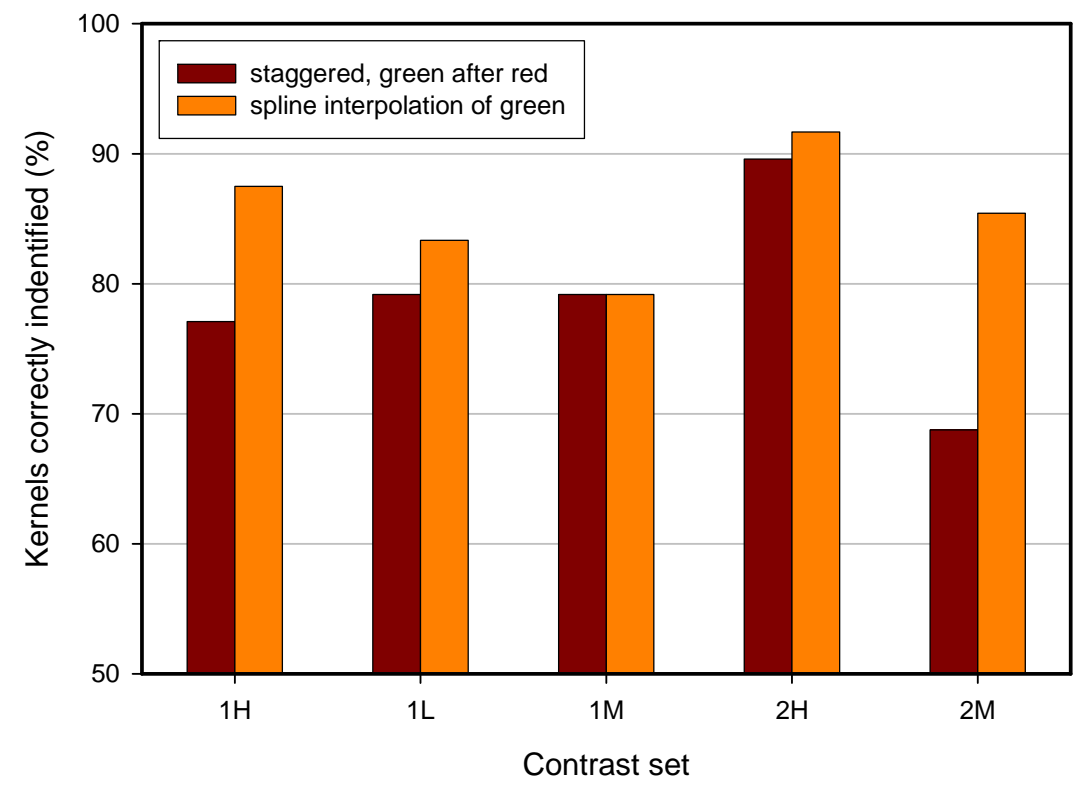

Figure 13 Comparison of the method of LED response pairing in linear discriminant analysis using the green-onto-red slope as the classification parameter, at the $45^{\circ}$ probe position. The two methods refer to the manner in which the green response is paired with the red response (stagger = green half-cycle response paired to preceding red half-cycle, spline interpolation of green = green responses adjusted by spline interpolation to time instances of red responses). 


\section{Method of Pairing LED Responses}

The two methods (staggered and interpolated) of pairing the LED response readings, used to determine the slope of the relationship between the two LEDs, are compared for the probe angle position of $45^{\circ}$ in Figure 13. The total percent correctly classified kernels from the resubstitution of the LDA function for each method are plotted for each contrast set. In four of the five sets, the spline interpolated method was superior to the staggered method. In the fifth set (moderate contrast), the classification rate was equal between the two methods. For the three other probe angle positions (not shown), the spline interpolation method was superior in all contrast sets.

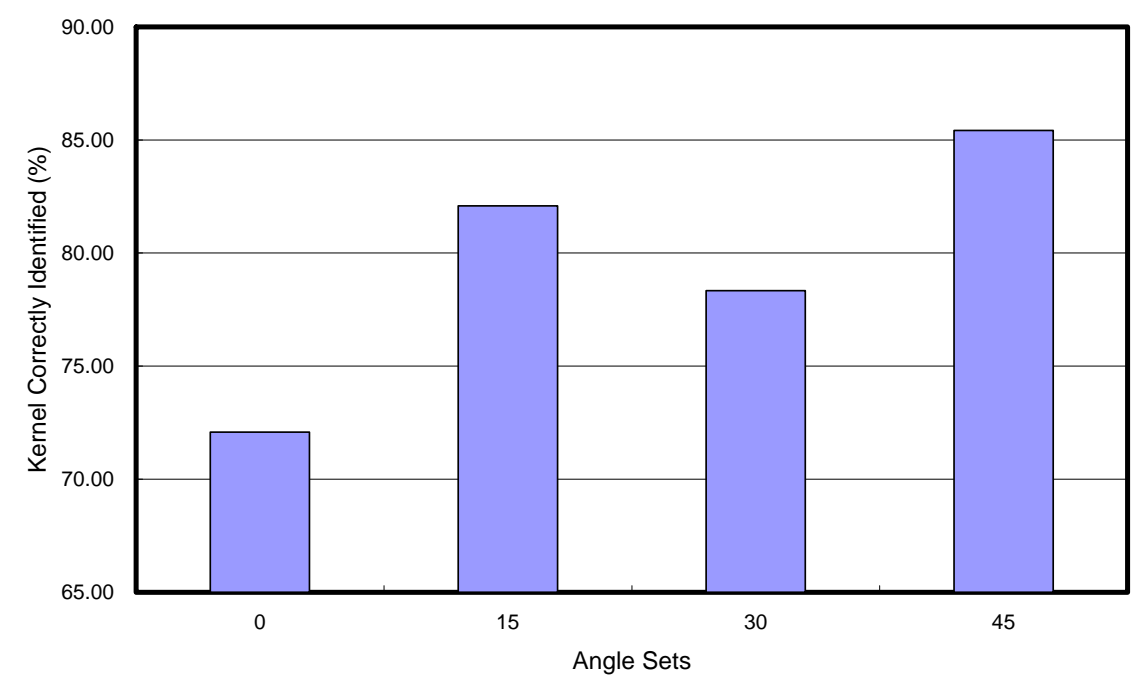

Figure 14 Percentage of kernels correctly identified at each measurement angle (analyzed by the optimal slope threshold method).

\section{Discussion}

Previous studies reported that color, rather than texture, as sensed by the free-falling kernel apparatus, was the dominant classifier. This finding was reaffirmed in the present analysis, as the coefficient of determination offered no significant contribution as a classifier. The two new findings of this study are both encouraging from the standpoint of improving the accuracy of recognition of Fusarium-damaged kernels in free-fall. The first finding is that placement of the probe at an oblique angle, such as $45^{\circ}$, enhances the reflected energy signal of both red and green LEDs, but more importantly, it also improves the overall accuracy, from $77 \%$ to $91 \%$ (Fig. 14). When the system was initially designed, the placement of the probe at the $0^{\circ}$ position was made for the purpose of reducing the relative contribution of surface reflectance compared to diffuse reflectance. Contrary to this original thought that the diffuse component would be more useful in separating normal and damaged kernels, it appears that the opposite idea is true - that surface color, rather than any underlying property, is the best parameter for identifying Fusarium-damaged kernels.

The second finding is that it is advantageous to adjust one of the LED responses (green was selected for this study) to the same time window as the other LED. A two-LED-one-detector system requires that the raw signal responses to be one-half cycle out of phase. The earlier 
assumption that a 0.25 -ms phase imbalance would be inconsequential to the classification models has been shown to be untrue. Instead, the interpolation scheme, either by cubic spline function (reported herein) or a simple linear interpolation (also tested but not reported), is beneficial to improving classification. This is because the regression analysis that relates the two LED responses is more accurately representing the responses to a common time basis, which was found to be critical in the use of slope as a classification parameter.

Lastly, while the bulk of this study concentrated on the clustering of kernel responses one sample at a time, we also explored the effect of angle when the kernels from all five samples are pooled together (Fig. 15). Again, the benefit of the oblique probe angle is apparent, with the $45^{\circ}$ angle demonstrating an optimal slope threshold level that correctly categorizes $85.4 \%$ of the kernels (Table 3 ), as compared with a low of $72.1 \%$ accuracy for the $0^{\circ}$ setting.

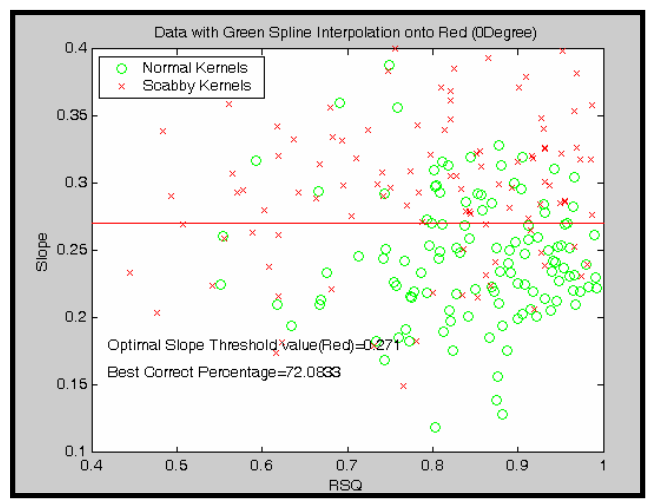

(A)

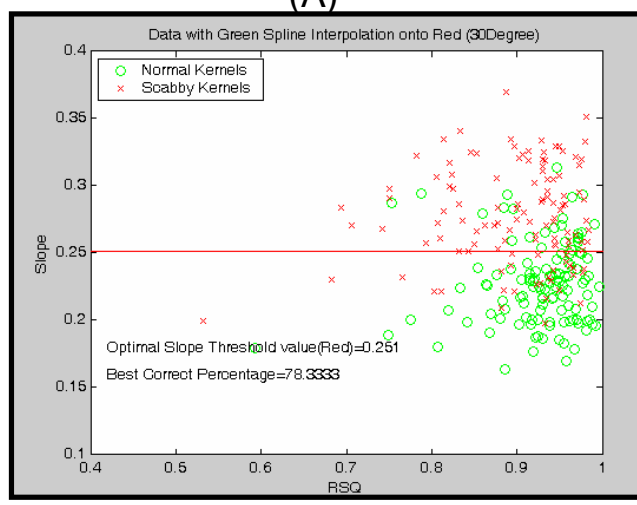

(C)

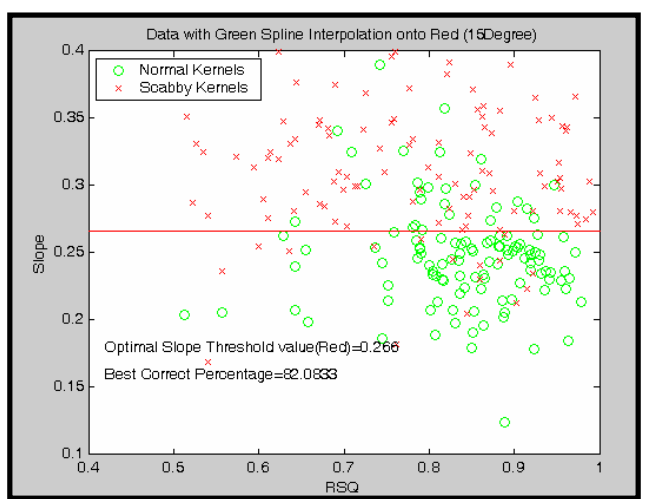

(B)

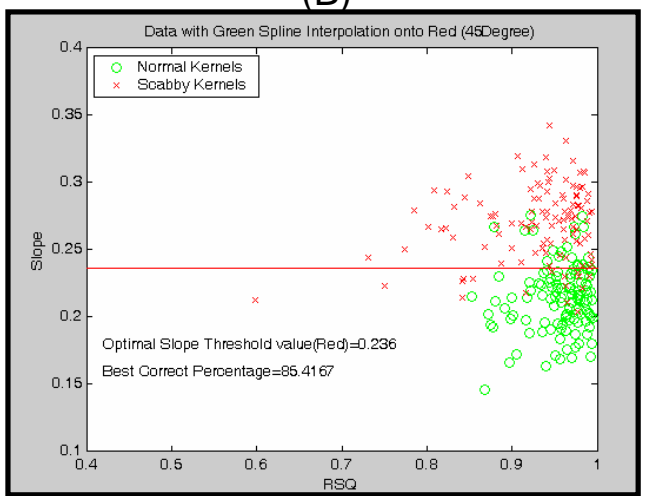

(D)

Figure 15 The RSQ and slope distribution for kernel samples of all contrast sets combined, and the optimal threshold for the combined set, for measurement angle of $(A) 0$ degree, (B) 15 degree, (C) 30 degree, (D) 45 degree.

Table 3 Results of optimal slope threshold method for the four measurement angles.

\begin{tabular}{|c|c|c|c|c|c|c|c|c|}
\hline \multirow{2}{*}{ Angle } & \multirow{2}{*}{ Contrast Level } & \multirow{2}{*}{$\begin{array}{c}\text { Slope } \\
\text { Threshold }\end{array}$} & \multicolumn{2}{|c|}{$\begin{array}{l}\text { Correctly Identified } \\
\text { Scab }\end{array}$} & \multicolumn{2}{|c|}{$\begin{array}{l}\text { Correctly Identified } \\
\text { Normal }\end{array}$} & \multicolumn{2}{|c|}{ Total Correct } \\
\hline & & & Out of 120 & (\%) & Out of 120 & (\%) & Out of 240 & (\%) \\
\hline 0 & \multirow{4}{*}{$\begin{array}{l}\text { Optimal } \\
\text { Threshold }\end{array}$} & 0.271 & 82 & 68.33 & 91 & 75.83 & 173 & 72.08 \\
\hline 15 & & 0.266 & 103 & 85.83 & 94 & 78.33 & 197 & 82.08 \\
\hline 30 & & 0.251 & 92 & 76.67 & 96 & 80.00 & 188 & 78.33 \\
\hline 45 & & 0.236 & 104 & 86.67 & 101 & 84.17 & 205 & 85.42 \\
\hline
\end{tabular}




\section{Conclusion}

The accuracy of the free-falling wheat kernel inspection system was enhanced by modifying the optical structure and the data analysis method. The angular position of the optical fiber with respect to the free-falling kernel and LED light source was modified to allow for acquisition of more reflected energy from the kernel. Of the three new angles examined $\left(15^{\circ}, 30^{\circ}\right.$, and $\left.45^{\circ}\right)$, the best result was obtained at 45 degrees. The spline interpolation was also used to calculate the simultaneous time axis data of red and green reflectance instead of a staggered calculation. The slope of the red and green regression function continued to be of greater utility as a classification parameter than the coefficient of determination. The best discrimination result of any single tray was $93.75 \%$, and the best discriminant result of all trays combined was $85.42 \%$.

\section{Acknowledgements}

The authors thank Professor Carl Griffey, Department Crop and Soil Environmental Sciences, Virginia Polytechnic Institute and State University, who contributed the Fusarium-damaged wheat samples. The authors also thank Miss Diane E. Chan for proofreading assistance.

\section{References}

Atanasoff, D. 1920. Fusarium-blight (scab) of wheat and other cereals. J. Agr. Res. 20:1-32.

Codex. 2003. Discussion paper on deoxynivalenol. Codex Committee on Food Additives and Contaminants, $35^{\text {th }}$ Session (Arusha, Tanzania, March 17-21, 2003). Codex Alimentaruis Commission, FAO/WHO, Rome, Italy.

Dowell, F.E., Ram, M.S., and Seitz, L.M. 1999. Predicting scab, vomitoxin, and ergosterol in single wheat kernels using near-infrared spectroscopy. Cereal Chem. 76: 573-576.

Dowell, F.E., Boratynski, T.N., Ykema, R.E., Dowdy, A.K., and Staten, R.T. 2002. Use of optical sorting to detect wheat kernels infected with Tilletia indica. Plant Dis. 86: 1011-1013.

Delwiche, S.R. 2003. Classification of scab- and other mold-damaged wheat kernels by nearinfrared reflectance spectroscopy. Trans. ASAE 46: 731-738.

Delwiche, S.R., and Hareland, G.A. 2004. Detection of scab damaged hard red spring wheat kernels by near-infrared reflectance. Cereal Chem. 81: 643-649.

Delwiche, S.R., and Gaines, C.S. 2005a. Wavelength selection for monochromatic and bichromatic sorting of Fusarium-damaged wheat. Appl. Eng. Agric. 21: 681-688.

Delwiche, S.R., Pearson, T.C., and Brabec, D.L. 2005b. High-speed optical sorting of soft wheat for reduction of deoxynivalenol. Plant Disease 89: 1214-1219.

Delwiche, S.R. 2008. High-speed bichromatic inspection of wheat kernels for mold and color class using high-power pulsed LEDs. Sens. Instrumen. Food Qual. (in press).

Dexter, J.E., and Nowicki, T.W. 2003. Safety assurance and quality assurance issues associated with Fusarium head blight in wheat. Pages 420-460 in: Fusarium Head Blight of Wheat and Barley. K.J. Leonard and W.R. Bushnell, eds. American Phytopathological Society, St. Paul, MN.

FAO. 2004. Worldwide regulations for mycotoxins in food and feed in 2003. Food and Nutrition Paper 81, Food and Agriculture Organization of the United Nations, Rome, Italy.

Hart, L.P. 1998. Variability of vomitoxin in truckloads of wheat in a wheat scab epidemic year. Plant Dis. 82: 625-630.

Luo, X., Jayas, D.S., and Symons, S.J. 1999. Identification of damaged kernels in wheat using a colour machine vision system. J. Cereal Sci. 30: 49-59. 
Pasikatan, M.C., and Dowell, F.E. 2003. Evaluation of a high-speed color sorter for segregation of red and white wheat. Appl. Eng. Agric. 19: 33-38.

Pasikatan, M.C., and Dowell, F.E. 2004 High-speed NIR segregation of high- and low- protein single wheat seeds. Cereal Chem. 81: 145-150.

Paul, P.A., Lipps, P.E., and Madden, L.V. 2005. Relationship between visual estimates of Fusarium head blight intensity and deoxynivalenol accumulation in harvested wheat grain: a meta-analysis. Phytopathology 95: 1225-1236.

Pearson, T.C., Wicklow, D.T., and Pasikatan, M.C. 2004. Reduction of aflatoxin and fumonisin contamination in yellow corn by high-speed dual wavelength sorting. Cereal Chem. 81: 490-498.

Ruan, R., Ning, S., Song, A., Ning, A., Jones, R., and Chen, P. 1998. Estimation of Fusarium scab in wheat using machine vision and a neural network. Cereal Chem. 75: 455-459.

SAS Institute Inc. 2004. Chapter 25. The DISCRIM Procedure. Pages 1137-1246 in: SAS/STAT® 9.1 User's Guide. : SAS Institute Inc., Cary, NC.

Stack, R.W. 2003. History of Fusarium head blight with emphasis on North America. Pages 1-34 in: Fusarium Head Blight of Wheat and Barley. K.J. Leonard and W.R. Bushnell, eds. The American Phytopathological Society, St. Paul, MN.

U.S. Food and Drug Administration. 1993. Letter from Ronald Chesemore to State Agricultural Directors, State Feed Control Officials, and Food, Feed and Grain Trade Organizations on Advisory Levels for DON (vomitoxin) in Food and Feed. U. S. Department of Health and Human Services, Public Health Service. September $16^{\text {th }}$, Rockville, MD. 